\title{
Laser heating of thick layers through the backwards, self-sustained propagation of a steep and steady state thermal front
}

\author{
M. Bruel ${ }^{\mathrm{a}}$ \\ Aplinov, Presvert No. 9, 38113 Veurey-Voroize, France
}

Received: 26 September 2008 / Received in final form: 30 October 2008 / Accepted: 9 December 2008 Published online: 25 December 2008 - (C) EDP Sciences

\begin{abstract}
We investigate theoretically a mode of heating layers using a laser beam. In this mode thermal energy, the temperature of the layer, propagates in a steady-state self sustained fashion from the bottom of the layer towards the surface and may exhibit a very steep front. The propagation exhibits a constant speed, related to the intensity of the power flux. To achieve this heating mode the absorption coefficient has to remain low in low temperatures and increase rapidly as a function of temperature in higher temperatures. Additionally, a significant temperature increase must be generated to trigger this propagation mode, for example through the presence of a strongly absorbing layer beneath the transparent layer. The mode is well suited to semiconductors, especially silicon. A few examples of applications are proposed.
\end{abstract}

PACS. 81.40.gh Other heat and thermomechanical treatments - 81.40.tv Optical and dielectric properties - 81.40.wx Radiation treatment

\section{Introduction}

Laser processing of materials has been studied and used for many years [1]. Powerful laser beams have been used with semiconductors as a tool for many different applications, such as annealing of implanted layers, alloying and etching $[2,3]$.

The primary effect of applying a laser pulse to a material is heating.

Laser energy is provided to the bulk material through the material's surface, leading to a profile of deposited energy peaking at the surface and decreasing with increasing depth. If the material is homogeneous and the absorption coefficient does not depend on temperature, the profile of this decrease is exponential.

The thermal energy profile arising from this laser flux is governed by the so called Fick laws. The profile is timedependant, decreases with increasing depth, and its extension within the bulk material evolves approximately following the square root of time.

In this paper, we present a theoretical evaluation of a new approach using a laser flux to heat up material layers, even very thick layers (several tens of microns). This evaluation is then confirmed by numerical simulation. It is required that the layer material does not absorb the chosen wavelength at the background temperature. Secondly, it is required that the absorption coefficient increases rapidly as a function of temperature in a range of higher temperatures. In these conditions, it will be shown

\footnotetext{
a e-mail: michel.bruel@aplinov.com
}

that a self-sustained propagation of a thermal front with a steady-state shape is possible, and that the propagation occurs at a constant velocity (in case of constant power flux) from the depth towards the surface. In addition, the initial conditions required to trigger this phenomenon are shown, so that after a transient period the steady state propagation is reached.

After the introduction, the second section of this paper covers the basic thermal aspects of a moving heat source. The third section shows that a heat source whose movement is self-sustained can be created inside a material and the fourth section presents a discussion on the limits of this theoretical work. The fifth section shows numerical simulations based on the conclusions of the first four sections, and we conclude with the sixth section, devoted to possible applications.

In this paper, the temperatures will be referenced to the background temperature $T_{0}$, for example, $T$ stands for $T-T_{0}$.

\section{Thermal aspects in case of moving heat sources}

The theory of moving heat sources and associated steady thermal regimes have been long studied by different authors such as Rosenthal [4] and Carslaw and Jaeger [5]. More recent theories have also been developed by Erhard [6] and Modest [7]. These theories generally deal with solving the heat equation in a semi-infinite solid, with a heat flux onto and through its surface that is moved by 


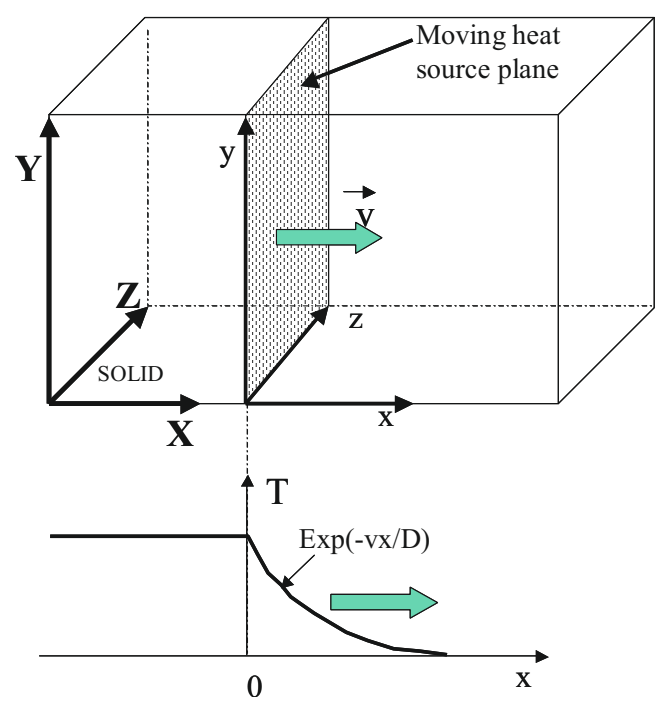

Fig. 1. (Color online) Schematics of the moving heat source plane.

external means. Some aspects of this theory are presented below.

We will consider an infinite homogeneous solid with rectangular co-ordinates $X Y Z$ bound to the solid (Fig. 1). This solid is subject to a moving heat source which generates heat over a plane perpendicular to the $x$ axis with a rate $\Phi$ (unit power per unit surface). The plane moves with velocity $v$ in the direction of the $x$ axis.

If we consider the rectangular co-ordinates $x y z$ referring to a fixed origin in the heat source plane and a given point within the solid, the relationship between the coordinates in the two systems are: $x=X-v t, y=Y$ and $z=Z$ with time origin taken when the two systems coincide.

The heat source can be represented by a Dirac delta function:

$$
S_{2 D}(x)=\Phi \delta(x) .
$$

It has been shown that in the moving $x y z$ co-ordinates a steady state temperature profile (shape independent of time) is reached [5]. This is described by the following set of relationships (Fig. 1):

$$
\left\{\begin{array}{l}
\text { For } x<0 T_{2 D}(x)=T_{\max } \\
\text { For } x>0 T_{2 D}(x)=T_{\max } \exp (-v x / D)
\end{array}\right.
$$

with $T_{\max }=\Phi / \rho C \nu$

where $\Phi$ is the incoming power flux, $\rho$ is the density, $C$ is the heat capacity, $D=k / \rho C$ is the thermal diffusion coefficient and $k$ is the thermal conductivity.

The profile consists of a plateau temperature and an exponential decrease.

Looking at the general case (steady state), the solid isn't submitted to a Dirac delta function $\delta(x)$, but is submitted to a heat source defined by $S(x)=\Phi s(x)$ where $s(x)$ is a physical function (approximately bell type) which is positive or null, continuous and whose integral equals to 1 .
From the previous set of relationships (2), further referring to the $T_{2 D}$ function, one can derive the relationship between $T$ and $x$ for any kind of heat source.

Let us remember that $s(x)$ can be considered as the convolution product of itself by the Dirac delta function.

$$
\begin{gathered}
s(x)=s(x) \otimes \delta(x) \\
\Phi s(x)=s(x) \otimes(\Phi \delta(x)) .
\end{gathered}
$$

As the heat equation is linear and as we have chosen constant coefficients, the relationship $T(x)$ between the temperature and $x$ coordinate is the convolution product of $s(x)$ and the $T_{2 D}(x)$ function:

$$
\begin{aligned}
T(x)= & s(x) \otimes T_{2 D}(x) \\
\Leftrightarrow & T(x)=\int_{-\infty}^{\infty} s(u) T_{2 D}(x-u) d u \\
\Leftrightarrow & T(x)=\int_{-\infty}^{x} s(u) \exp (-v(x-u) / D) d u+\int_{x}^{\infty} s(u) d u .
\end{aligned}
$$

It can be seen that $T(x)$ is the sum of two components.

$\int_{X}^{\infty} s(u) d u$ is the adiabatic part, the relationship between $T(x)$ and $s(x)$, when there is no heat diffusion within the solid $(D=0)$.

For example, in the case of a Gaussian distribution, $s(x)$ is described by the following relationship:

$$
s_{\text {Gauss }}(x)=\left(1 /\left(X_{0} \sqrt{2 \pi}\right)\right) \exp \left(-x^{2} / 2 X_{0}^{2}\right)
$$

which leads to:

$$
\begin{aligned}
T_{\text {Gauss }}(x)= & \left(\left(1 / X_{0} \sqrt{2 \pi}\right)\right. \\
& \left.\times \exp \left(-x^{2} / 2 X_{0}^{2}\right)\right) \otimes T_{2 D}(x) \\
T_{\text {Gauss }} / T_{\max }= & \left(1 / X_{0} \sqrt{2 \pi}\right) \int_{-\infty}^{x} \exp \left(-u^{2} / 2 X_{0}^{2}\right) \\
& \times \exp (-\nu(x-u) / D) d u \\
& +\left(1 / X_{0} \sqrt{2 \pi}\right) \int_{x}^{\infty} \exp \left(-u^{2} / 2 X_{0}^{2}\right) d u
\end{aligned}
$$

with the reduced variables: $\delta=x /\left(X_{0} \sqrt{2 \pi}\right), \eta=(D / v) /$ $\left(X_{0} \sqrt{2 \pi}\right), \Theta_{\text {Gauss }}=T_{\text {Gauss }} / T_{\max }$ one gets

$$
\begin{aligned}
\Theta_{\text {Gauss }}= & (1 / 2) \operatorname{erfc}(\delta \sqrt{\pi})+(1 / 2) \exp \left(1 / 4 \pi \eta^{2}\right) \\
& \times \exp (-\delta / \eta) \operatorname{erfc}(-\delta \sqrt{\pi}+1 / 2 \eta \sqrt{\pi}),
\end{aligned}
$$

$X_{0} \sqrt{2 \pi}$ represents the width of the source profile, defined as the width of a rectangular profile whose height equals 


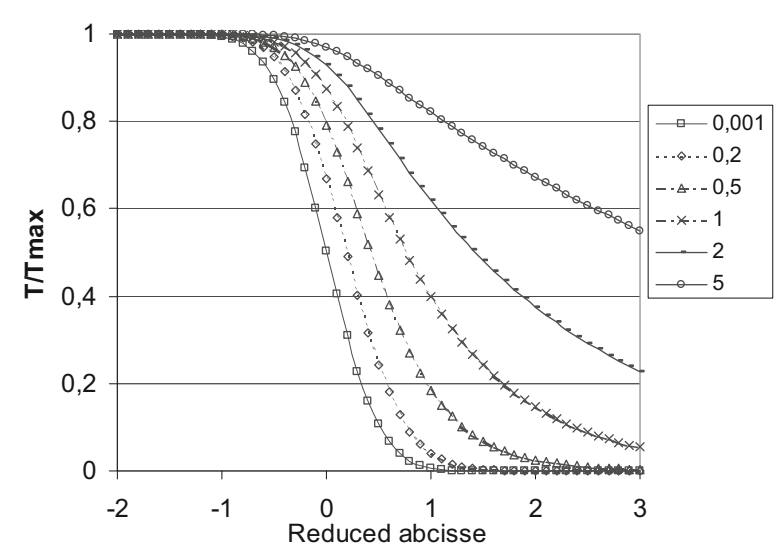

Fig. 2. Temperature profile as a function of the reduced abcisse in the moving coordinates system. For the Gaussian source profile, for different values of $\eta$.

the peak value of the actual source profile. $D / v$ is homogeneous to a length and is an important parameter in all moving source issues. The $\eta$ parameter defines the ratio between the length $D / v$ and the width of the source profile.

Figure 2 illustrates the evolution of $\Theta_{\text {Gauss }}(\delta, \eta)$ with $\eta$ as parameter. The shape is sigmoidal; the lower parameter $\eta$ (the smaller $D / v$ compared to the width $X_{0} \sqrt{2 \pi}$ ), the closer the temperature curve is to the deposited energy curve and the shorter the distance which is needed to reach the plateau temperature.

\section{How to create a moving thermal source within the solid}

The next question is how to create, within the bulk of a solid, a thermal source extending in a steady-state bell shape fashion on both sides of a plane moving with respect to the solid, at a constant speed?

The first approach is to imagine a way of creating a power flux from the outside of the solid, which can penetrate the solid and is able to deposit energy in a concentrated fashion at a given depth, changing the outside flux parameters in order to modify this energy deposition depth.

A rough approximation could be to use energetic particles with small mass, such as protons, which deposit most of their energy near the end of penetration range of the particles and subsequently generate a concentrated thermal energy around the corresponding depth.

Varying the particle velocity varies the depth at which the particles create this thermal source. However, this approach is only a rough approximation and is difficult to implement, especially if one needs to change the particle velocity very rapidly.

The second approach, developed in this paper, consists of a situation where the power flux is still created from a source outside the material but the movement of the thermal source, the evolution of the depth at which the power flux is transformed into thermal energy, is no longer governed from outside the solid but is governed by the actual thermal source. In other words, in combination with the incoming flux, the thermal source provides the mechanism which moves the thermal source. The movement of the thermal source inside the solid is self-sustained.

Let's consider a photon flux $\Phi$ coming from the right side which penetrates a homogeneous solid with an absorption coefficient $\alpha$; the power flux is absorbed according to the relationships:

$$
\begin{aligned}
& d \Phi(X) / \Phi(X)=\alpha d X \\
& \Leftrightarrow \Phi(X)=\Phi\left(X_{a}\right) \exp \left(\alpha\left(X-X_{a}\right)\right) \\
& \Leftrightarrow \Phi(X-v t)=\Phi\left(X_{a}-\nu t\right) \exp \left(\alpha\left(X-X_{a}\right)\right) \\
& \Leftrightarrow \Phi(x)=\Phi\left(x_{a}\right) \exp \left(\alpha\left(x-x_{a}\right)\right) .
\end{aligned}
$$

The relationship between $\Phi$ end coordinates is the same in both systems of coordinates $X Y Z$ and $x y z$.

$\alpha$ depends on temperature, especially in semiconductors. As a result, one has to write:

$$
\begin{gathered}
d \Phi(x) / \Phi(x)=\alpha(T(x)) d x \\
\Leftrightarrow \alpha(T(x))=(d \Phi(x) / d x) / \Phi(x) \\
\text { with }\left\{\begin{array}{c}
S(x)=d \Phi / d x \\
\Leftrightarrow \Phi_{0}=\int_{-\infty}^{+\infty} S(u) d u \\
\Phi(x)=\Phi_{0}-\int_{x}^{\infty} S(u) d u=\int_{-\infty}^{x} S(u) d u \\
\alpha(T(x))=S(x) \int_{-\infty}^{x} S(u) d u=s(x) / \int_{-\infty}^{x} s(u) d u .
\end{array}\right.
\end{gathered}
$$

Associating equation (17) with equation (5) provides a relationship between $\alpha$ and $T$.

$$
\left\{\begin{aligned}
T(x) & =s(x) \otimes T_{2 D}(x) \\
\alpha(T(x)) & =s(x) / \int_{x}^{-\infty} s(u) d u
\end{aligned}\right.
$$

For example: let's consider the heat source whose shape (Fig. 3) is described by the following set of equations:

$$
s_{\text {comp }}(x)=\left\{\begin{aligned}
\left(1 / X_{\text {eq }}\right) & \exp \left[\begin{array}{l}
-\left(x+X_{0} / 2\right)^{2} /\left(2 X_{\text {left }}^{2}\right. \\
\left.\times\left(1+\left(x+X_{0} / 2\right) / X_{\text {lin }}\right)\right)
\end{array}\right] \\
& \text { for } x<-X_{0} / 2 \\
\left(1 / X_{\text {eq }}\right) \times 1 & \text { (18a) } \\
& \text { for }-X_{0} / 2<=x<=X_{0} / 2 \quad(18 \mathrm{~b}) \\
\left(1 / X_{\text {eq }}\right) & \exp \left(-\left(x-X_{0} / 2\right) / X_{\text {right }}\right. \\
& \text { for } X>X_{0} / 2
\end{aligned}\right.
$$

where $X_{e q}$ is set so that the integral of $s(x)=1$. 


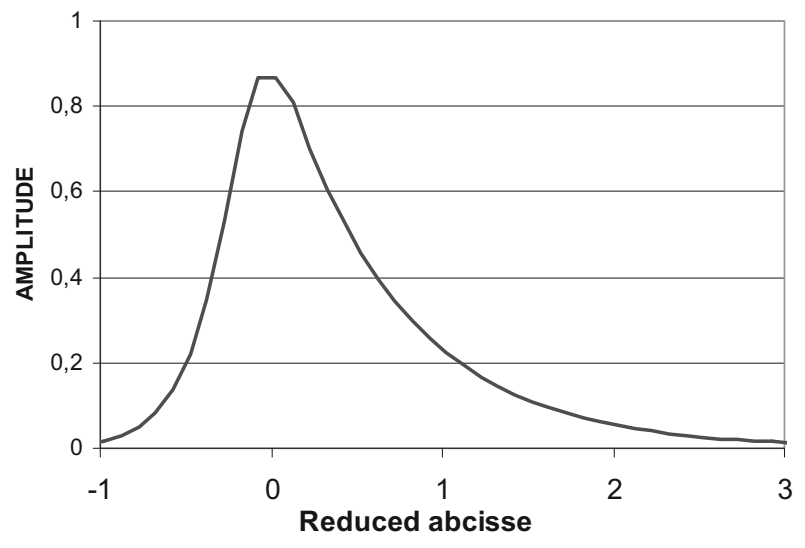

Fig. 3. Profile of the composite heat source defined in equation (18), corresponding to the parameters: $k_{\text {right }}=0.7, k_{0}=0.15$, $k_{\text {left }}=0.15$ and $k_{\text {lin }}=0.25$.

The heat source composite profile is a continuous curve with a plateau surrounded by an exponential decrease on the right and a Gaussian curve ending as an exponential decrease on the left. It is possible to independently choose $X_{0}, X_{\text {left }}, X_{\text {right }}$ and $X_{\text {lin }}$ allowing different shapes.

We will name $T_{\text {comp }}$ the temperature in the case of this composite profile.

$$
T_{\text {comp }}(x)=s_{\text {comp }}(x) \otimes T_{2 D}(x) .
$$

This convolution product can be established either analytically or by numerical calculation.

The following reduced variables are now used:

$$
\begin{aligned}
\delta & =x / X_{e q}, k_{\text {right }}=X_{\text {right }} / X_{\text {eq }}, k_{\text {left }}=X_{\text {left }} / X_{\text {eq }}, \\
k_{0} & =X_{0} / X_{e q}, k_{\text {lin }}=X_{\text {lin }} / X_{e q} \\
\eta & =(D / v) / X_{e q}, \Theta_{\text {comp }}=T_{\text {comp }} / T_{\text {max }} .
\end{aligned}
$$

As defined in the former section, $X_{e q}$ represents the width of the source profile and $\eta$ the ratio between $D / v$ and the width of the source profile.

Figure 4 displays the relationship between $\Theta_{\text {comp }}$ and the reduced abcisse $\delta$. In this example $k_{\text {right }}=0.7, k_{0}=$ $0.15, k_{\text {left }}=0.15, k_{\text {lin }}=0.25 \eta$ varying between 0 and 50 .

Figure 5 shows the relationships between absorption coefficient $\alpha$ and the temperature.

The shapes of these curves show an increase of $\alpha$ with the temperature; one can also see that the smaller $\eta$ is, the more rapid the increase.

Coming back to the interpretation of the relationship between $\alpha$ and $T$, we consider the curve $\alpha(T)$ of Figure 5 for $\eta=0.5$, referring to it as $\alpha_{0.5}(T)$.

Taking a material with density $\rho$ heat capacity $C$ and whose absorption for a given wavelength as a function of temperature can be described by the relationship $\alpha_{0.5}(T)$, we can irradiate this material through one of its faces with a light flux of the aforementioned wavelength with a power flux $\Phi$.

If good initial conditions are chosen, a steady state and self-sustained moving thermal regime will develop with a

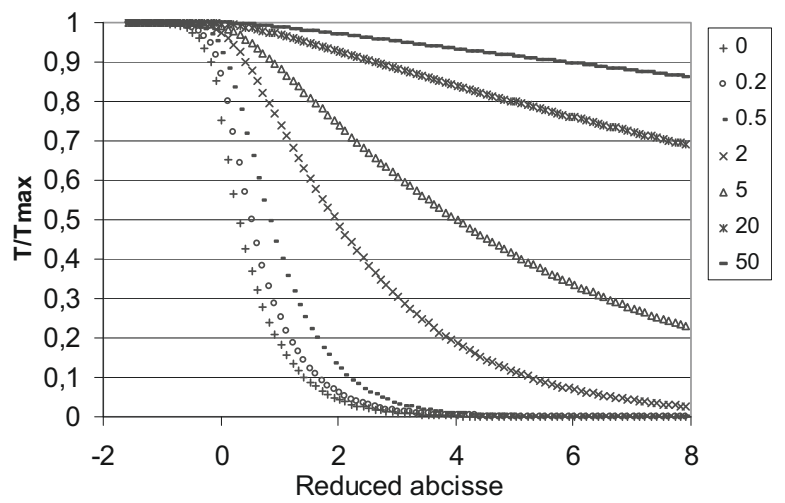

Fig. 4. Temperature profile as a function of the reduced abcisse in the moving coordinates system for the composite source profile corresponding to the set of parameters: $k_{\text {right }}=0.7$, $k_{0}=0.15, k_{\text {left }}=0.15$ and $k_{\text {lin }}=0.25 . \eta$ is the parameter.

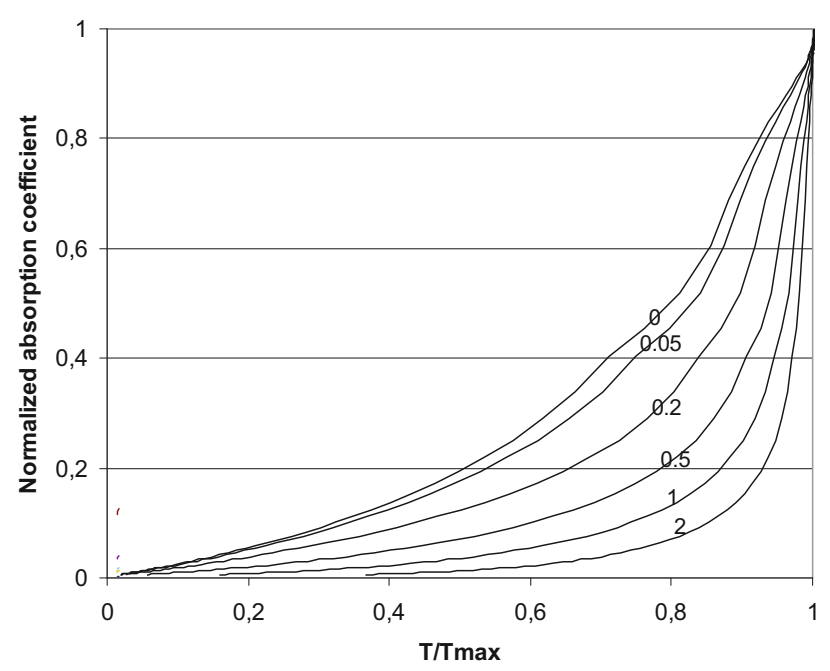

Fig. 5. Relationship between absorption coefficient (normalized to 1) and temperature extracted from equations (17) and (5) for the composite profile with the previous quoted set of parameters. $\eta$ is the parameter.

heat front whose shape is shown in Figure 4, corresponding to $\eta=0.5$, moving towards the left side with a velocity $v$, given by the equation $\eta=(D / \nu) / X_{e q}=0.5$, and where the maximum temperature is given by $T_{\max }=\Phi / \rho C v$.

It must be pointed out that as long as the flux $\Phi$ remains, the movement of the thermal front is self-sustained.

\section{Discussion}

Here, we have shown that a self-sustained, steady-state and steep heat front can exist and propagate at a constant speed in the opposite direction to an incoming power flux.This propagation mode is possible provided 
that the absorption coefficient is an increasing function of temperature.

In addition, it must be stressed that the absorption coefficient is zero for $T=0$. This is obvious when looking at equation (17). It becomes

$$
\alpha(T=0)=s(+\infty) / \int_{-\infty}^{\infty} s(u) d u=s(+\infty) / 1
$$

$s(\infty)$ must equal 0 , otherwise the integral $\int_{-\infty}^{\infty} s(u) d u$ would diverge.

As a result the pure ideal backwards and self-sustained propagation of a steep thermal front cannot exist in an actual physical situation, because it is physically unlikely that an absorption coefficient could strictly equal 0 at $T=T_{0}$.

Nevertheless, there are lots of physical cases which approximate the theoretical conditions so that it is possible to obtain backwards and self-sustained propagation of a steep thermal front with a quasi steady-state shape. This requires the existence of a temperature range at and above the background temperature where the absorption coefficient remains low. For a given experiment, the theoretical evaluation of the temperature increase at the surface at the end of the projected experiment, can provide a good criterion. The lower this temperature increase at the surface, compared with the expected plateau temperature, the closer the thermal profile evolution is to the theoretical case.

The transient period, the duration necessary to reach the steady-state propagation, and the creation of conditions leading to a steady-state propagation after a transient period are also key issues from a physical point of view.

In an infinite solid whose absorption coefficient is zero at its background temperature, nothing happens when irradiated by the power flux $\Phi$ - neither a steady-state heat front propagation, nor a temperature increase.

A solution is to create a layer called the triggering layer at a given depth, whose initial temperature is well above background temperature so that it is absorbing, or to create a layer where the absorption coefficient is not equal to zero at the background temperature. In both cases, the incoming flux will be absorbed by this triggering layer and will gradually transfer part of its thermal energy to the neighbouring material which in return will become more and more absorbing. The temperature profile will progressively change from the bell-shape (symmetric at $t=0$, and then more and more dissymmetric with an increasing tail) around the triggering layer to a moving steady-state steep thermal front moving at a constant speed towards the surface. The modelling of this transient state is highly complex and lies outside the scope of this paper. However, some general principles can be addressed, namely that the higher the initial temperature or absorption coefficient of the triggering layer, compared to the steady-state plateau temperature, the shorter the transient period and the smaller the distance for the temperature profile to be in a steady state, measured from the triggering layer upwards.

\section{Simulation of silicon heating with a $\mathrm{CO}_{2}$ laser}

The evolution of the thermal profile in bulk silicon irradiated through one of its faces by a $\mathrm{CO}_{2}$ laser beam was simulated. The wavelength $(10.6 \mu \mathrm{m})$ of this kind of laser is suitable because the absorption coefficient at room temperature in un-doped silicon is around $2 \mathrm{~cm}^{-1}\left(\alpha_{L}=\right.$ lattice absorption) and is dependent on the temperature because its major contribution is the free carriers' absorption.

The absorption coefficient can be described by [8]:

$$
\alpha(T)=\sigma(T)(N d+N i(T))+\alpha_{L}
$$

where

$$
\sigma(T)=1.9 \times 10^{-20} T^{3 / 2} \mathrm{~cm}^{2} .
$$

The intrinsic density $N i(T)$ is given by:

$$
N i(T)=n_{0} T^{3 / 2} \exp \left(-E_{g} / k T\right)
$$

The variation of the gap energy with the temperature must be taken into account, it can be approximated by:

$$
E_{g}=1.17-\left(4.73 \times 10^{-4} T^{2}\right) /(T+636) .
$$

Figure 6 shows the steep variation of the absorption coefficient as a function of temperature, calculated using equations 21 to 24 . It can be observed that there is a domain in the vicinity of the background temperature where the absorption coefficient remains quite low. Also, there is a domain of higher temperatures with a very strong increase.

The structural example which was chosen is essentially a highly doped $\left(5 \mathrm{E} 19 \mathrm{~cm}^{-3}\right)$ silicon substrate on top of which a very thick layer $(150 \mu \mathrm{m}$ thick), doped to $1 \mathrm{E} 15 \mathrm{~cm}^{-3}$, was grown. The role of the highly doped substrate is to operate as a trigger, as explained in the earlier section.

Computer software was written to solve the one dimensional heat equation based upon the finite differences method. This was used to simulate the evolution of the thermal profile. In these simulations, variations of heat capacity and thermal conductivity with temperature were not taken into account; constant values $\left(1 \mathrm{~J} / \mathrm{g}{ }^{\circ} \mathrm{C}\right.$ for heat capacity, $0.5 \mathrm{~W} / \mathrm{cm}^{\circ} \mathrm{C}$ for thermal conductivity) were chosen. However, this simplification does not change the quality of the results.

The incoming flux (it is coming from the left side ) was fixed at $2 \times 10^{6} \mathrm{~W} \mathrm{~cm}^{-2}$ and the duration to $20 \mu \mathrm{s}$. The background temperature being chosen at $300 \mathrm{~K}$.

These results are presented in Figure 7; the temperature profile within the solid is represented at increasing times with step increases of $2 \mu \mathrm{s}$. The timing starts when the flux $\Phi$ is switched on. By looking at the results, we can see that the steady-state regime is obtained after a 


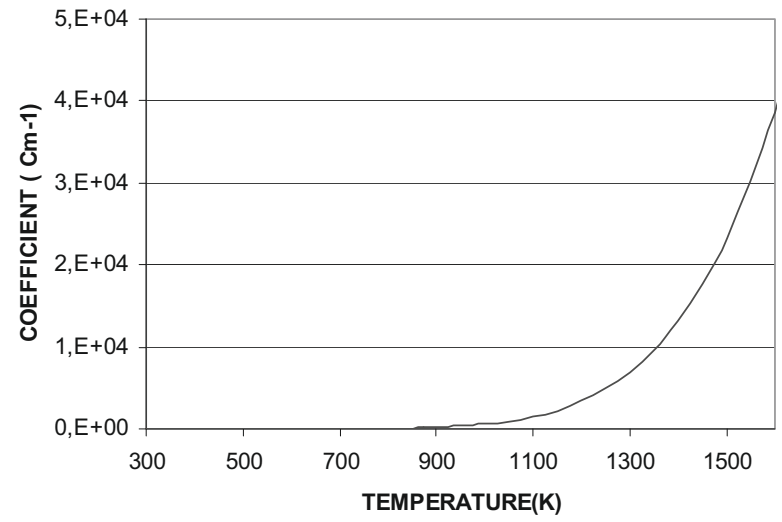

(a)

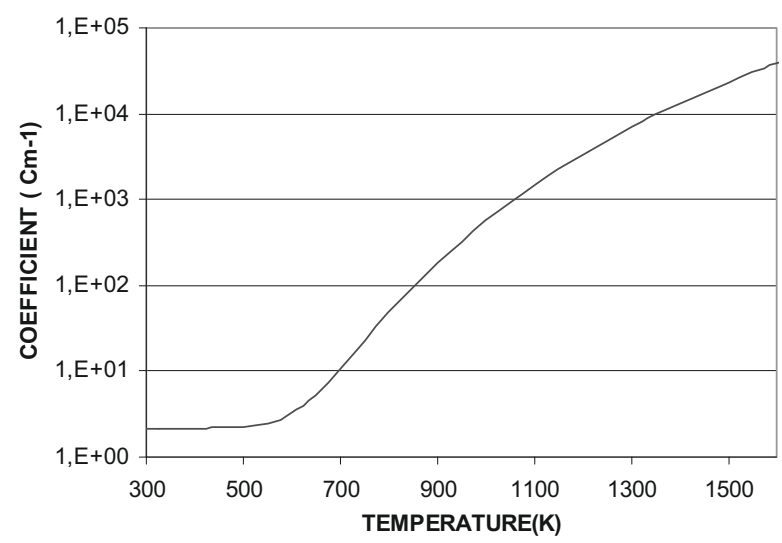

(b)

Fig. 6. (a) Absorption coefficient of silicon versus temperature at the $10.6 \mu \mathrm{m}$ wavelength (linear scale). (b) Absorption coefficient of silicon versus temperature at the $10.6 \mu \mathrm{m}$ wavelength (logarithmic scale).

transient period of about $4 \mu \mathrm{s}$. This is very similar to the steady-state regime described in the previous sections and exhibits a progression speed of $7 \mu \mathrm{m} / \mu \mathrm{s}(700 \mathrm{~m} / \mathrm{s})$ with a plateau temperature of $1476 \mathrm{~K}$, which corresponds to a temperature increase of $1176 \mathrm{~K}$.

We can also see the gradient of the front. In the linear section, the temperature rises from $600 \mathrm{~K}$ to $1400 \mathrm{~K}$ in a distance of approximately $4.5 \mu \mathrm{m}$.

Inserting numerical values of $T_{\max }, C, \rho$ and $v$ into equation (2c) confirms that the values from the simulation are coherent with the model:

$$
\Phi / \rho c v=2 \times 10^{6} /(2.43 \times 1 \times 700)=1176=T_{\max } .
$$

\section{Applications}

Three main characteristics of this method for heating layers must be emphasized: suitability for heating very thick layers, highly homogeneous heating throughought the thickness of the layer, the very fast process (in the range of ten tenths of a microsecond to some microsec-

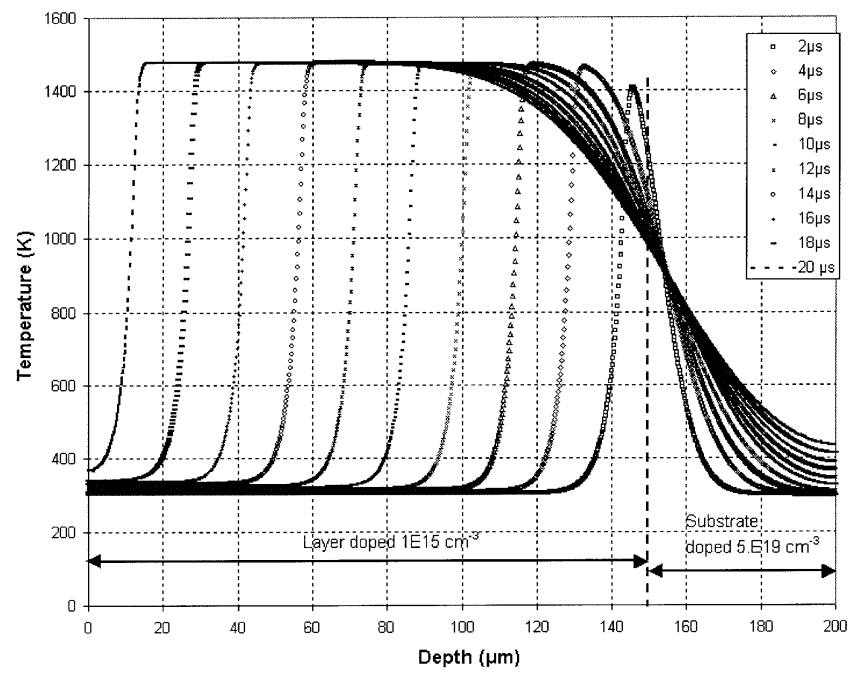

Fig. 7. Numerical simulation of the evolution of the temperature profile of the structure defined in Section 5 by a laser flux of $2 \mathrm{E} 6 \mathrm{~W} / \mathrm{cm}^{2}$ at the wavelength of $10.6 \mu \mathrm{m}$. The profile is represented at time intervals of $2 \mu \mathrm{s}$.

onds) and quasi adiabatic heating of the layer (almost no heating of underlying substrate).

In the previous section, an example was given using a $\mathrm{CO}_{2}$ laser beam at a wavelength of around ten microns. Other wavelengths can also work, especially those whose absorption by free carriers is the main mechanism, so that the absorption coefficient depends highly on the temperature.

In the case of single crystal-silicon (low doped) for processing layers of medium thickness (for example, 20 microns), YAG lasers or Ytterbium doped fibre lasers at around 1.06-1.08 microns are suitable, provided that they can provide energy pulses of the required power and duration. They are less well adapted to very large thicknesses because of the magnitude of the absorption coefficient at room temperature, lying between $20 \mathrm{~cm}^{-1}$ and $300 \mathrm{~cm}^{-1}$, depending on the authors. Using low background temperatures such as LN2 could improve the situation by reducing the band gap height, and thus reducing near band edge absorption. Erbium doped fibre lasers at around $1.5 \mu \mathrm{m}$ and other fibre lasers at around 2 and $3 \mu \mathrm{m}$ are interesting for single-crystal silicon, but would be particularly interesting for the heat treating of poly crystal materials, as absorption at the YAG wavelength could be too high, especially in the case of polysilicon.

It is also possible to melt layers. One can melt silicon either across the entire thickness of the layer or across a portion of the layer thickness. Melting a material buried under a surface could be used for different applications, for example to segregate impurities in a deep region without affecting the surface layer or as a method to improve the crystalline quality of a buried layer by melting it and letting the re-solidification process proceed by epitaxial regrowth using the un-melted top-layer as the template.

Melting an entire layer could be useful to re-crystallise non single-crystal layers, either epitaxially or not, in order 
to get either single-crystal layers or layers with larger grains.

This could be useful in the field of photovoltaic materials.

\section{Conclusion}

It has been demonstrated that it is possible to heat thick (from micrometers to several tens) layers in a quasi adiabatic fashion in a microsecond range at a very homogeneous plateau temperature.

Some experimental work is currently underway to assess this new concept.

The existence of high power fibre lasers and their further developments are also of great interest for applications based upon this new concept.

Thanks to P. Mottier for establishing the core of the finite differences software in 2003. Thanks to Philip Ewels for improving the english.

\section{References}

1. J.D. Majumdar, I. Manna, Laser processing of materials, Vol. 28 (Indian Academy of Sciences, Sadhana, 2003), pp. 495-562

2. R.T. Young, R.F. Wood, Ann. Rev. Mater. Sci. 12, 323 (1982)

3. P. Baeri, E. Rimini, Mater. Chem. Phys. 46, 169 (1996)

4. D. Rosenthal, Trans. Am. Soc. Mech. Eng. 68, 849 (1946)

5. H.S. Carslaw, J.C. Jaeger, Conduction of heat in solids, 2nd edn. (Oxford Science Publications, 1959), p. 510

6. P. Erhard, C. Hölle, C. Karcher, Int. J. Heat. Mass Transfer 36, 3997 (1993)

7. M.F. Modest, H. Abakians, Trans. Am. Soc. Mech. Eng. 108, 598 (1986)

8. M. Sheik-bahae, H.S. Kwok, J. Appl. Phys. 63, 518 (1988)

To access this journal online: www.edpsciences.org 